\title{
The Improvement of CSMA/CA Based on Dynamic Weighting Algorithm
}

\author{
Yuexing $\mathrm{Gu}$ \\ Qingdao University of Science and Technology, Shandong 266061, China \\ gyx870421@163.com
}

Keywords: Wireless sensor network (WSN); IEEE802.15.4; CSMA/CA; Dynamic weighting algorithm; Heavy load.

\begin{abstract}
IEEE802.15.4 defines MAC and PHY specification for low-rate wireless personal area networks (LR-WPANS), which targets wireless networking among low-rate, low-power and low-cost devices for short range telecommunication. As the wireless sensor network (WSN) used in ward monitoring system need to handle large amounts of data, this paper proposes an improved back-off algorithm in slotted CSMA/CA by introducing a weighting algorithm of NB. By NS2 simulation, when the traffic load is large, compared with IEEE 802.15.4, the delay time, packet loss rate and Jitter of the improved dynamic algorithm can be significantly reduced, and the throughput of the improved dynamic algorithm can be significantly increased. In this paper, the improved CSMA/CA algorithm can dynamically change as the changes of the traffic load, and take different back-off measure to suit the situation. Besides, the improved CSMA/CA algorithm not only keep the superiority of the standard CSMA/CA algorithm in small traffic load, but also can achieve reliable transmission of data in real time under heavy load conditions.
\end{abstract}

\section{Introduction}

The wireless sensor network is the integrated application of sensor technology, wireless communication technology and network protocol, which brings new development trend to the existing computer network. The wireless sensor network is tend to be applied in areas which contain large amount of data and be complex, such as ward monitoring system. But there are many problems varying from design to application need to be solved ${ }^{[1]}$. Among the large number of short distance communication protocols IEEE 802.15.4 is the most commonly used one for WPAN(Wireless Personal Area Network ). The protocol, whose important targets are low energy, low transmission rate and low cost, developed a physical layer and a MAC layer specification to provide uniform standards for low speed interconnections between individual or home ranges ${ }^{[2]}$.However, as the wireless sensor network becomes more complex and contains larger amount of data ,new requirements are proposed. This paper improved the IEEE 802.15.4 protocol to make packets transmission more reliable and efficient.

At present, the research on the performance of the QOS of the wireless sensor network is mainly in the condition of the limited energy to improve the real-time and reliability of the network ${ }^{[3-4]}$. At present, there are a series of research on the multi carrier monitoring (CSMA/CA) algorithm with the collision avoidance of IEEE802.15.4. The paper ${ }^{[5]}$ analysis the performance of slotted CSMA/CA algorithm in the contention access period.A new power/rate scheduling mechanism based on the CSMA/CA is proposed in the paper ${ }^{[6]}$, aiming at improving the throughput of the self-organized network while increasing the transmission rate. The new analysis model of CSMA/CA algorithm for IEEE802.15.4 is proposed in the saturation conditionfrom the throughput and energy consumption of the two aspects in the paper ${ }^{[7]}$.A practical service differentiation mechanism is proposed and discussed to improve the performance of the time slot CSMA/CA for the related event by improving the 802.15.4 IEEE protocol in the paper ${ }^{[8]}$.the Markoff analysis model of CSMA/CA algorithm in IEEE 802.15.4 is presented in the unsaturated state in the paper ${ }^{[9]}$.A discrete time version of the CSMA algorithm is proposed in the paper ${ }^{[10]}$, and the collision free transmission schedule is explicitly considered during the protocol control. More importantly, this algorithm can allow us to guarantee the optimal throughput while getting smaller delay. In the literature ${ }^{[11]}$, it is based on the modified 
backoff exponent BE for further improvement of IEEE802.15.4, and analysisfrom the throughput and energy consumption.

According to the high amount of data in the network environment (for example ward monitoring system) in the specific requirements, This paper improves the IEEE802.15.4 protocol, which makes the 802.15.4 protocol more suitable for the dynamic of network in real application. The weighted algorithm and the original CSMA/CA backoff algorithm for the fusion of IEEE 802.15.4 protocol, so as to make improvements. We sum the previous transmission by weighted sum,to select the appropriate CSMA/CA index BE backoff algorithm, so we can determine the appropriate backoff period, get the optimal performance of the network. We use NS2 to backoff algorithm of dynamic weighting of CSMA/CA based on simulation. It is found that the improved protocol can adapt to network dynamic change.

\section{IEEE 802.15.4 MAC Layer Introduction}

IEEE 802.15.4 defines the physical layer and MAC layer specification, we consider only the MAC layer. The protocol supports two topologies: star structure and point structure, in a star network, two kinds of communication: beacon-enabled and non-beacon enabled, this paper considers the beacon-enabled mode of star network .

\subsection{Super-Frame Structure}

In beacon-enabled PAN must be used in the super-frame structure, and is a beacon frame indicating the start of a superframe. Superframe generated and sent by the coordinator superframe parameters are determined by the coordinator. Superframe separated from each other by beacon frames, and is divided into 16 equal time slots. Within the first slot is located in the super frame beacon frame for synchronization problems connected devices in order to set up a PAN. In the active period, and it can be divided into competitive access period CAP and non-competitive access period CFP.In competitive access period CAP, all devices must CSMA / CA mechanism to contend for the channel, enabling transmission of data, but the transmission of all data must be completed before the end of the CAP.

Some of the delay for data demanding or require specific transport, PAN coordinator in a part of the space will be allocated for this part of the specific needs of data transmission, and part of the PAN is to ensure that the slot allocation GTS.GTS guaranteed slot is located in the inactive period CFP, in the super-frame structure followed by the competitive access period CAP, as shown in FIG 1. In the 16 slots superframe, the coordinator can assign up to seven slots for guaranteed slots GTS, can at least 0 time slots, while a guaranteed slot GTS can occupy multiple slots. GTS slots in ensuring the transmission of data, you must own the allocated time slot or to complete the transfer of data before the end of CFP. However, in the active phase of the CAP, it allows all network devices or even neighboring networks to re-apply to join the network of all devices by CSMA / CA channel access mechanism competition.

As shown in Figure 1, the length of the superframe BO is controlled by BI, and the length of the active period SD controlled by the SO. Equation as follows:

$$
\begin{aligned}
& B I=\text { aBaseSuperframeDuration } * 2^{B O} \quad 0 \leq B O \leq 14 \\
& S D=\text { aBasesSuperframeDuration } * 2^{\text {so }} \quad 0 \leq S O \leq B O \leq 14
\end{aligned}
$$

Among them, the parameters BO, SO is determined by PIB of the MAC layer and notify other nodes via beacon frame. Different from literature [14], this article does not consider the inactive state of the work, not taking into account to ensure the slot GTS and use of the acknowledgment frame $\mathrm{ACK}$, so we make $\mathrm{BO}=\mathrm{SO}$. Finally, in order to reduce complexity, we do not consider frame interval IFS in analysis and simulation stage of the algorithm.

\subsection{CSMA/CA Algorithm}

In the active period of super-frame structure, all nodes can transmit packets in two ways, in particular, in the phase of the competitive access period CAP, all the nodes can access mechanism by 
CSMA / CA channel, and in non-competitive access of CFP, only the appointment of a node can be achieved by ensuring data transmission slot GTS, so as to achieve the purpose of collision avoidance.

In CSMA with time slot / CA algorithm, all nodes are required before access channel for time synchronization with the PAN, twice channel detection CCA, and determine the three parameters (NB, BE, CW) values. Wherein, NB is the number of back-off, showing the current competitive access procedure backoff times, NB initial value is set to 0 , then once every backoff, NB value plus 1 ; $\mathrm{BE}$ is backoff index, each channel determines the detection performed (CCA) nodes backoff time before backoff time, BE minimum represented by macMinBE; $\mathrm{CW}$ is the contention window, indicate the need for channel CCA (clear channel assessment) the number of detection before transferring data, the initial value of 2 , CCA once successfully decreased 1 , when the $\mathrm{CW}$ is 0 can transmit data. CSMA / CA algorithm specific processes with time slots as shown below:

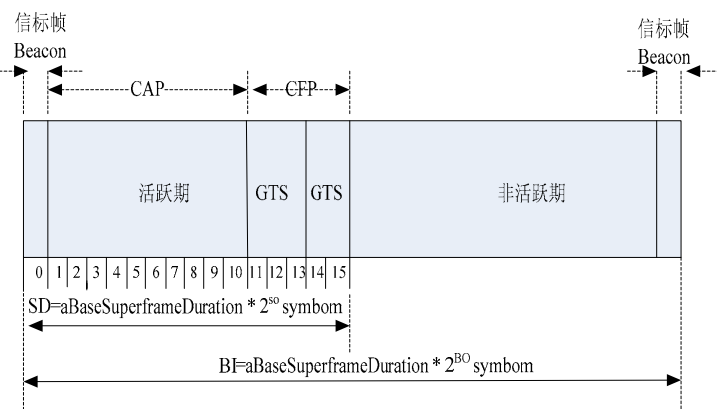

Figure 1 Super Frame Structure

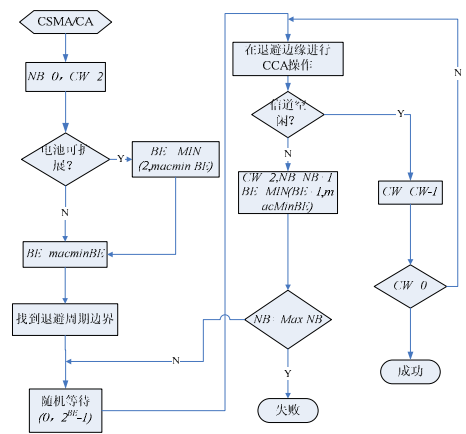

Figure 2 CSMA/CA Algorithm

\section{Introduction of CSMA/CA Algorithm based on Dynamic Weighting}

\subsection{Question}

Wireless sensor network protocol in IEEE 802.15.4 is a low rate, low power consumption, low equipment cost of short distance communication one. However, in recent years, wireless sensor networks have more and more applications in the areas with a relatively large amount of data, e.g.: IEEE 802.15.4 protocol used in ward monitoring system has appeared more problems. These problems are mainly in the following several aspects: 1) the amount of network data changes, and whether the original protocol can meet the dynamic changes of network or not;2)the amount of network data relative to traditional wireless area one is large, and whether the original protocol can provide a relatively stable, efficient transmission environment for the large amount of data or not; 3 ) in the completion of the transmission of large amounts of data, and whether the network performance can be guaranteed or not.

For the above three aspects proposed, some improvements of the IEEE 802.15.4 protocol with collision avoidance CSMA/CA backoff algorithm were made. The improvement idea is based on the dynamic weighting algorithm and the standard CSMA/CA algorithm, and the CSMA/CA algorithm based on dynamic weighting was proposed. The protocol will better meet the network needs by improving the backoff algorithm parameters include the exponential backoff, contention window and retreat times.

\subsection{Improved CSMA/CA Algorithm based on Dynamic Weighting}

The range of change of backoff algorithm parameters of CSMA/CA algorithm in IEEE 802.15.4 protocol is (macMinBE, aMaxBE), the macMinBE is defined as the minimum value of backoff exponent, in the range between 0 and 3 , and the default value 3 . The value range of $a M a x B E$ generally is 3 to 8 , the default value is 5 , and the initial value of backoff times NB is 0 , the maximum value $\max N B$ is 5. In the CSMA/CA Algorithm based on Dynamic Weighting proposed in this paper, the minimum value of $\mathrm{BE}$ is set to 1 , the contention window $(\mathrm{CW})$ is set to 2 , and the maximum number of backoff is set 5 .

Considering different network dynamics and node transmission conditions, the paper introduced NB dynamic weighting algorithm to choose the backoff exponent BE by comparing the weighting value of NB and its value. When the value of BE decreases, the backoff period $0 \sim 2^{B E-1}$ will decreases, 
which makes it possible for nodes to backoff shorter time to access channel and this application in channel contention access to a relatively small number of nodes or a smaller volume of business; on the contrary, the backoff period $0 \sim 2^{B E-1}$ will increases, which males nodes need backoff for relatively long time to access channel, and the application in channel contention access a larger number of nodes or the large amount of business. The concrete implementation programme is as follows:

When NB is larger than weighted value of $\mathrm{NB} \overline{N B}$, namely $N B>\overline{N B}$, or the number of nodes increases, increasing the minimum value of $\mathrm{BE}$, namely macMin $B E=L B E+1$, thereby increases the value of a backoff period;

When NB is smaller than weighted value of $\mathrm{NB} \overline{N B}$, namely $N B<\overline{N B}$, or the number of nodes decreases, keeping the minimum value of $\mathrm{BE}$, namely macMin $B E=L B E-1$, thereby decreases the value of a backoff period;

If the above two cases do not match with each other, the parameter settings are same as the last transmission.

The parameters NB mentioned above are the data transmitted successfully when the node accesses to the channel last time, namely the documented numerical; the weighted values of NB $\overline{N B}$ is the weighting of the resulting numerical according to first five times value of NB; LBE indicated the value of $\mathrm{BE}$ when the node successfully access to the channel to achieve data transmission. When the node successfully accesses to channel, packet are transmitted, and records the value of NB; When node failed accesses to channel and records the value of NB, then the value of NB is the maximum value of NB $\operatorname{Max} N B$. The formula of calculating the weighted value of NB $\overline{N B}$ is as follows:

$$
\overline{N B}=a_{1} * N B_{i-4}+a_{2} * N B_{i-3}+a_{3} * N B_{i-2}+a_{4} * N B_{i-1}+a_{5} * N B_{i}, \text { where } \sum_{i=1}^{5} a_{i}=1
$$

Where $i$ indicates the times of accessing to the channel; $a_{1}, a_{2}, a_{3}, a_{4}, a_{5}$ are respectively weighted coefficients and should meet with the requirements $a_{1} \leq a_{2} \leq a_{3} \leq a_{4} \leq a_{5}$, for the reason that the value of NB's effect will change a lot in the $i$ access to the channel with the value of $i ; N B_{i}, N B_{i-1}, N B_{i-2}, N B_{i-3}, N B_{i-4}$ indicates respectively the value of NB in $i, i-1, i-2, i-3, i-4$ access to the channel; The flow of improved CSMA/CA algorithm based on dynamic weighting is as shown in Figure 3:

To improve CSMA/CA algorithm to better adapt to the changes in network load, the range change of the parameters of $\mathrm{BE}$ can be set larger in the paper, in which the macMinBE is set to 1, the maximum of $\mathrm{BE} a M a x B E$ is set to 8 , and the maximum number of MaxNB is set is 5 . For the selection of the weighted parameters, the paper has set the weighted coefficients of multiple simulation results as follows: $a_{1}=0.05, a_{2}=0.1, a_{3}=0.2, a_{4}=0.3, a_{5}=0.35$. Each group of weighted coefficients corresponding to the simulation results are shown in Table 1.

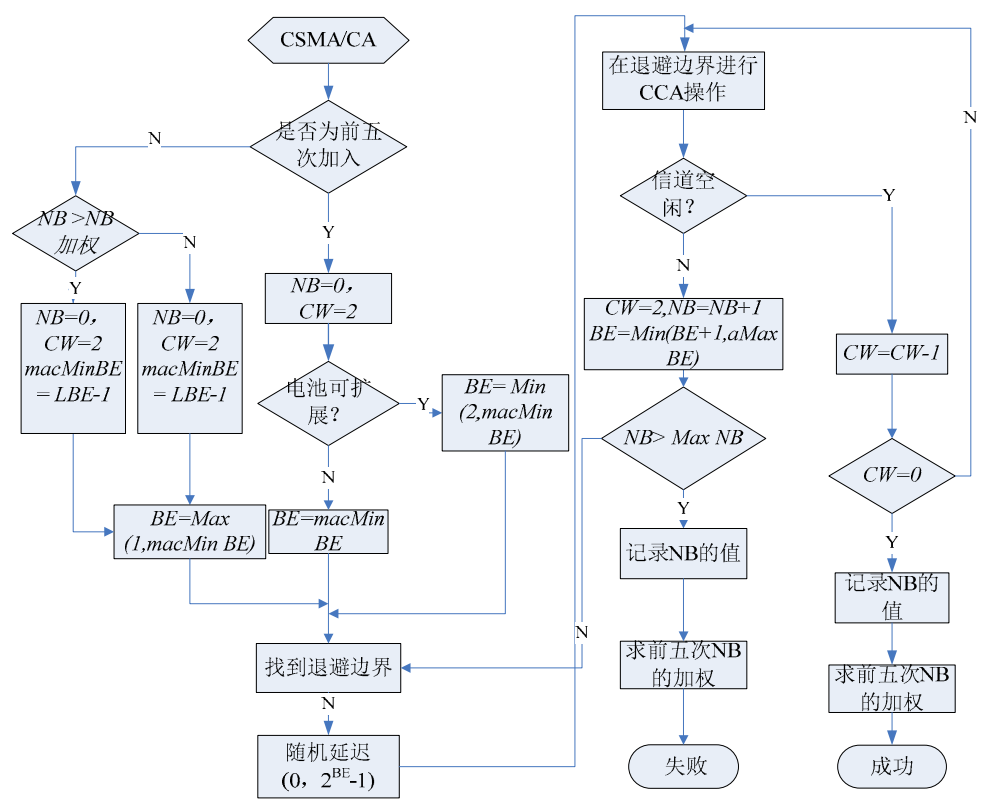


Figure 3 CSMA/CA Algorithm based on Dynamic Weighting 


\section{Simulation}

In order to verify the superiority of improved protocol, the paper adopted NS2 simulation to analyze the performance of improved 802.15.4 protocol, and compared the simulation results between the IEEE 802.15.4 protocol and improved IEEE 802.15.4 based backoff mechanism. Based on the improved backoff mechanism of IEEE $802.15 .4^{[13]}$, proposed an improved backoff algorithm based on time slot mechanism in the process of CSMA/CA. At the start of each CSMMCA, the minimum value of the contention window is dynamically adjusted according to the transmission condition of the past, and when the service load is relatively high or the packet size is relatively small, it can reduce the probability of packet collision and retransmission probability, which can reduce the power consumption and improve the throughput performance. Simulation parameters are set as shown in Table 2. In the simulation environment of this paper, the network is set for a star topology with 25 nodes, of which only a node is a coordination function point, the remaining 24 ones are ordinary ones and only the uplink communication is considered. All nodes are fixed ones, and are in the communication range of each other, without taking the problem of the hidden terminal into account.

The wireless coverage with a range of network for $50 \mathrm{~m} * 50 \mathrm{~m}$ is selected and the network performance parameters simulation analysis is carried out according to the load changing from $10 \mathrm{kbps}-100 \mathrm{kbps}$ through the simulation time of $100 \mathrm{~s}$.

In the following analysis, all data are obtained from the average of 10 sets of experimental data, and the impact of $95 \%$ confidence interval is adopted, both the error of the random error and the simulation deviation are reduced. This chapter mainly analyzes the network performance from four aspects: transmission delay, packet loss rate, Jitter and network throughput. The network performance comparisons are mainly for CSMA/CA algorithm based on dynamic weighting in 802.15.4, the improved backoff mechanism based on IEEE 802.15.4 ${ }^{[11]}$, and the CSMA/CA algorithm in IEEE 802.15.4. In the following simulation graph, the paper uses IEEE802.15.4 to represent the CSMA/ CA algorithm of standard IEEE 802.15.4; uses 802.15.4 to represent the improvement mechanism of backoff algorithm based on IEEE 802.15.4; and uses improved 802.15.4 to represent the CSMA/CA algorithm based on dynamic weighting.

Table 1 Weighted Coefficient

\begin{tabular}{|c|c|c|c|c|}
\hline Weighted Coefficient & $\begin{array}{c}\text { Transmission } \\
\text { Delay } / \mathrm{s}\end{array}$ & Jitter & $\begin{array}{c}\text { Packet } \\
\text { Loss Rate }\end{array}$ & Throughput \\
\hline $\mathrm{a}_{1}=0.15, \mathrm{a}_{2}=0.2, \mathrm{a}_{3}=0.2, \mathrm{a}_{4}=0.2, \mathrm{a}_{5}=0.25$ & 0.052306 & 0.007184 & $21.7 \%$ & 38 \\
\hline $\mathrm{a}_{1}=0.15, \mathrm{a}_{2}=0.15, \mathrm{a}_{3}=0.2, \mathrm{a}_{4}=0.2, \mathrm{a}_{5}=0.3$ & 0.052407 & 0.007273 & $22.6 \%$ & 36 \\
\hline $\mathrm{a}_{1}=0.1, \mathrm{a}_{2}=0.2, \mathrm{a}_{3}=0.2, \mathrm{a}_{4}=0.25, \mathrm{a}_{5}=0.25$ & 0.049235 & 0.006215 & $24.9 \%$ & 40 \\
\hline $\mathrm{a}_{1}=0.1, \mathrm{a}_{2}=0.15, \mathrm{a}_{3}=0.2, \mathrm{a}_{4}=0.25, \mathrm{a}_{5}=0.3$ & 0.049898 & 0.006172 & $26.5 \%$ & 46 \\
\hline $\mathrm{a}_{1}=0.05, \mathrm{a}_{2}=0.1, \mathrm{a}_{3}=0.2, \mathrm{a}_{4}=0.3, \mathrm{a}_{5}=0.35$ & 0.048871 & 0.005946 & $17.6 \%$ & 52 \\
\hline $\mathrm{a}_{1}=0, \mathrm{a}_{2}=0.05, \mathrm{a}_{3}=0.15, \mathrm{a}_{4}=0.3, \mathrm{a}_{5}=0.5$ & 0.050217 & 0.006389 & 18.6 & 48 \\
\hline
\end{tabular}


Table 2 Simulation Parameters

\begin{tabular}{cc}
\hline Parameters & Values \\
\hline Network range & $50 \mathrm{~m} * 50 \mathrm{~m}$ \\
Simulation Time & $100 \mathrm{~s}$ \\
Node Number & 25 \\
Load & $10 \sim 100 \mathrm{kps}$ \\
Topological Type & Star Network \\
Route Type & AODV \\
$B O, S O$ & $B O=3 . S O=3$ \\
\hline
\end{tabular}

\subsection{Transmission Delay}

The transmission delay refers to the time when the packet arrives from the start and the data packet is successfully sent. The size of the transmission delay indicates the real-time performance of the network. Figure 4 shows the different traffic load influence on the delay by respectively using the three algorithms when (CSMA/CA algorithm based on dynamic weighting, improvement mechanism of backoff algorithm based on IEEE 802.15.4,CSMA/CA algorithm in IEEE 802.15.4) traffic load and, where the time delay is the average delay time.

As can be seen from Figure 4, with the increase of the amount of network traffic, the transmission delay is increasing, and the incremental range is also increased. When the traffic load is between $10 \mathrm{kbps}$ and $20 \mathrm{kbps}$, all the transmission delay of three algorithms keeps constant. When the traffic load is between $20 \mathrm{kbps}$ and $100 \mathrm{kbps}$, all the three transmission delay increases sequentially. The transmission delay is decreased from delay 3 to delay 1 , and the incremental range of the transmission delay is increased from delay3, delay 2 to delay1. By analyzing the transmission delay above, when the traffic load is small, the difference of three algorithms of transmission delay is small, and tends to be stable; when the traffic load is high, delay 3 is far greater than delay 2 and delay 2 is far greater than delay1. Thus, when the traffic load is relatively large, the CSMA/CA algorithm based on dynamic weighting proposed in this paper has a greater advantage than the other two algorithms.

\subsection{Jitter}

Packets must waiting in the queue of nodes for sending because of the changing of network, the flow is sometimes large and sometimes small, so the transmission time for each packet is different, the differences is called Jitter. The bigger the Jitter, the more unstable the network is. Figure 5 shows the changes of the Jitter in the simulation of three different algorithms.

As shown in fig 5, Jitter 3,the Jitter value of algorithm IEEE802.15.4, changes in the vicinity of 0.008; Jitter 2,the Jitter value of algorithm CSMA/CA ,changes in the vicinity of 0.00687 ; Jitter 1, the Jitter value of proposed algorithm CSMA/CA which is based on dynamic weighted, changes in the vicinity of 0.006 . As analysised, the Jitter values of three algorithms can keep stable in their respective range, however the proposed algorithm CSMA/CA which is based on dynamic weighted has a smaller change range when the traffic load changes, be more compatible with the changing of network and make packets be more reliable and stable transmission.

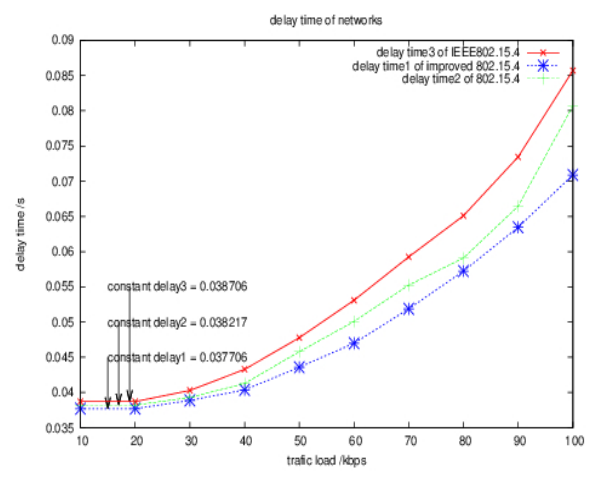

Figure 4 Traffic Load-Transimission Delay

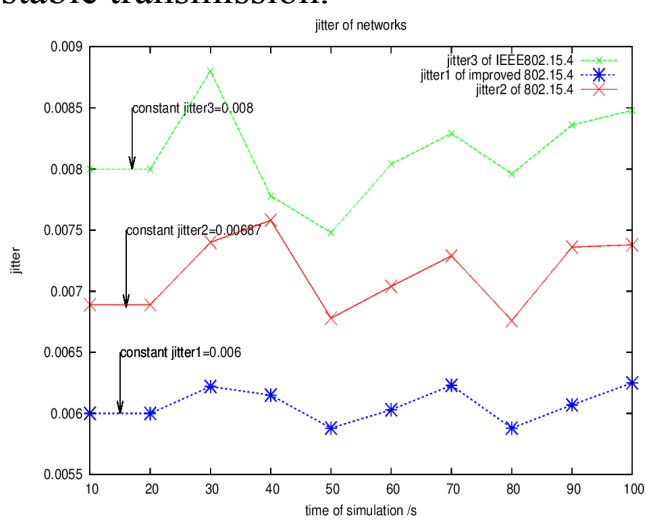

Figure 5 Changes of Jitter with Time 


\subsection{Packet Loss Rate Analysis}

Packet Loss Rate is the loss rate percentage of the total number of packets sent by the system in the process of testing. For the research of the packet loss rate, the throughput can be met. Figure 6 shows the impact of three algorithms on the packet loss rate.

As can be seen from Figure 6, when the traffic load is between $10 \mathrm{kbps}$ and 30kbps, packet loss rate of three algorithms remained the same. When the traffic load increases, packet loss rate of three algorithms also increases, and the one of CSMA/CA algorithm based on dynamic weighting is much smaller than the other two. Through the simulation data, when the traffic load of three algorithms increases, the packet loss rate of them and the differences between the three packet loss rate also increases. As for the analysis, the proposed CSMA/CA algorithm based on dynamic weighting has an advantage over the other two algorithms, and the more the traffic load is, and the bigger the advantage is. It comes to the conclusion that the proposed CSMA/CA algorithm based on dynamic weighting is more compliant to the situation that the traffic load is large.

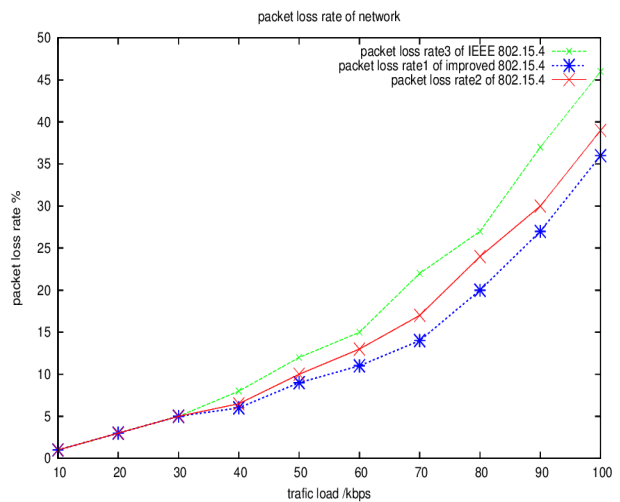

Figure 6 Anysis of Traffic Load -Packer Loss Rate

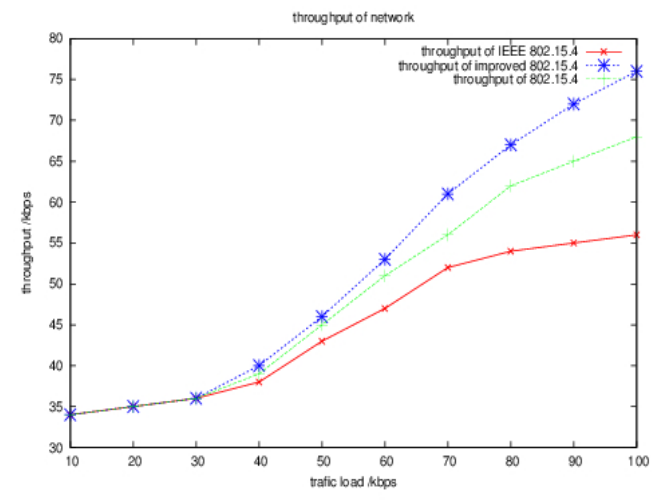

Figure 7 Anysis of Traffic Load -Throughput

\subsection{Throughput Analysis}

Network throughput testing refers to a test of network maintenance and trouble finding, especially analysis of the problem related to network performance. Through analysis of throughput, the performance of network equipment under different configuration can be obtained, and then the relevant settings are optimized and evaluated. Figure 7 showed that the traffic loads of three algorithms' effect on the throughput, in which the throughput is the average throughput of the whole network.

As can be seen from Figure 7, when the traffic volume changes between $10 \mathrm{kbps}$ and 30kbps, the corresponding throughput of three algorithms is basically the same. However, when the traffic volume changes between $30 \mathrm{kbps}$ and $100 \mathrm{kbps}$, the throughput of the three is increasing, and the throughput of the CSMA/CA algorithm based on the dynamic weighting is significantly higher than the other two algorithms. All the throughput of three algorithms increases with the volume of business increases properly, however, the volume of business increases to a certain extent, and it tends to be a stable state. But it cannot rule out that the business volume continued to increase in the process whether the throughput appear decreasing sharply (this is mainly due to the volume of traffic exceeds the network load that it can bear and the he system collapses). As the simulation results showed that, when the volume of business is larger, the throughput of CSMA/CA algorithm based on the dynamic weighting in 802.15.4 proposed in this paper are obviously much larger and other two ones, which has more advantages.

\section{Conclusions}

In this paper, the author considered dynamic weighting algorithm and backoff algorithm of CSMA /CA and proposed CSMA/CA algorithm based on dynamic weighting. CSMA/CA algorithm based on dynamic weighting is applied to 802.15.4 IEEE, which can make it adapt to the dynamic changes 
of the network. CSMA/CA algorithm based on dynamic weighting proposed in this paper can adjust macMinBE from 1 to 8 according to the dynamics between comparisons of the value $N B$ and weighted ones $\overline{N B}$ of $N B$. Simulation results validated our scheme, and improved delay, Jitter and packet loss rate and throughput are better with the original 802.15.4, and the paper found that in a business with relatively large load, this advantage is more obvious. The dynamic algorithm introduced reduced the network collision rate and retransmission rate, so that it has greater advantages in delay, Jitter, packet loss rate and throughput reflects.

\section{References}

[1] Emil Jovanovl, Aleksandar 1 lilmkoviel,Chri8 Otto,et a1. A wireless body area network of intelligent motion sensors for computer asisted physical rehabilitation [J/OL]. J Neuroeng Rehabil. 2005 Mar 1, 2(1):6.

[2] IEEE- TG15.4, "Part 15.4: Wireless Medium Access Control (MAC) and Physical Layer (PHY) Specifications for Low- Rate Wireless Personal Area Networks (LR-WPANs). IEEE standard for Information Technology, 2003

[3] Li Fangmin Coordination between Liu Xinhua power control techniques for wireless sensor networks [J] Journal of Software, 2008,19 (3): 716-732.

[4] Liu Yunlu, Pu Juhua, Fang Weiwei, Zhang Xiong wireless sensor network MAC protocol optimization algorithm [J] Journal of Computers, 2012, 3: 529-539.A. N. Alvi, S. S. Naqvi, S. H. Bouk, N. Javaid, U. Qasim, Z. A. Khan. Evaluation of Slotted CSMA/CA of IEEE 802.15.4 [J]. Broadband, Wireless Computing, Communication and Applications (BWCCA), 2012 Seventh International Conference on .12-14 Nov. 2012:391 - 396.

[5] Han-Chiuan Luo, Eric Hsiao-Kuang Wu, Member, IEEE, and Gen-Huey Chen. A Transmission Power/Rate Control Scheme in CSMA/CA-Based Wireless Ad Hoc Networks. [J]. Vehicular Technology, IEEE Transactions on (Volume: 62, Issue: 1).Jan. 2013:427 - 431.

[6] T.R. Park ,T.H. Kim ; J.Y. Choi ; S. Choi ; W.H. Kwon. Throughput and energy consumption analysis of IEEE 802.15.4 slotted CSMA/CA [J]. Electronics Letters,Volume41, Issue 18, 1 September 2005, p. $1017-1019$.

[7] Anis Koubaa, Mário Alves, Bilel Nefzi, Ye-Qiong Song. Improving the IEEE 802.15.4 Slotted CSMA/CA MAC for Time-Critical Events in Wireless Sensor Networks [J]. Technical Report. Jul 2006.

[8] Chang Yong Jung, Ho Young Hwang, Dan Keun Sung, Gang Uk Hwang, Enhanced Markov Chain Model and Throughput Analysis of the Slotted CSMA/CA for IEEE 802.15.4 Under Unsaturated Traffic Conditions [J]. Vehicular Technology, IEEE Transactions on (Volume: 58, Issue: 1) .Jan. 2009: 473 - 478.

[9] Jian Ni,Bo (Rambo) Tan,R. Srikant.Q-CSMA: Queue- Length-Based CSMA/CA Algorithms for Achieving Maximum Throughput and Low Delay in Wireless Networks [J].Networking, IEEE/ACM Transactions on (Volume: 20, Issue: 3).June 2012 :825 - 836.

[10]Jicheng, Xuyou Yun, ZHENG Bao-yu. 802.15.4 improved mechanism backoff algorithm [J]. Information Technology, 2008,8.J. Mi ic, V. B. Mi ic, and S. Shafi. "Performance of IEEE802.15.4 beacon enabled PAN with uplink transmis- -sions in non-saturation mode-access delay for finite buffers", IEEE BroadNets 2011, San Jose, CA,October 2004. 